\title{
Summation of transfer of adaptation gradients
}

\author{
CURTIS W. McINTYRE \\ Southern Methodist University, Dallas, Texas \\ DOUGLAS A. HARDWICK \\ Illinois State University, Normal, Illinois \\ and \\ HERBERT L. PICK, JR. \\ University of Minnesota, Minneapolis, Minnesota
}

\begin{abstract}
Transfer of adaptation resulting from a 90-deg rotation of the visual field was examined when movement during exposure was limited to either one or two dimensions. Positive transfer of adaptation was found for all conditions; however, the curvilinear pattern of adaptation for the one-dimension exposure condition was different from that found for the two-dimension exposure condition. Subsequent analyses revealed the pattern of adaptation obtained for the two-dimension exposure condition was similar to that predicted by an algebraic summation of the patterns associated with the one-dimension exposure conditions. These results indicated that the dimensions of perceptual-motor space are not organized as either an integrated system or as an independent set.
\end{abstract}

Research by McIntyre and Pick (1974) and Pick, Warren, McIntyre, and Appel(1972) has been directed toward establishing whether or not perceptual-motor space is an integrated system. Initially, two alternative conceptualizations were considered: Either perceptual-motor space is an integrated system of dimensions in which a modification of one dimension results in the whole system's being modified, or it is a system of independent dimensions in which a modification of one dimension is confined specifically to that dimension. The above authors' empirical results led them to conclude that neither of these alternatives was correct. Rather, using a transferof-adaptation paradigm, they found a curvilinear transfer function from a single dimension exposed during adaptation to targets along nonexposed dimensions. Specifically, in their experiments, subjects were exposed to a 90-deg rotation of the visual field. During exposure, subjects were constrained to move their hands back and forth along a linear track. The visual display of the track and movement was, of course, rotated by $90 \mathrm{deg}$. Adaptation was measured by noting the change from before to after exposure when subjects copied a line in the same visual orientation as the track. Transfer of adaptation was determined by measuring the changes

The present study was supported in part by the University of Minnesota Center for Reseach in Human Learning and by Institute of Child Development Program Project Grant HD-03082. The authors express their appreciation for the help of Allan Frank, Robin Palkovitz, and James Musselwhite in the collection of data. Requests for reprints should be sent to: Curtis W. McIntyre, Department of Psychology, Southern Methodist University, Dallas, Texas 75275. after exposure when subjects copied lines in other orientations. In particular, almost complete transfer of adaptation was found for target lines rotated $90 \mathrm{deg}$ from the original visual orientation of the track. Surprisingly, there was significantly greater than $100 \%$ transfer found for target lines lying intermediate (in the direction of rotation distortion) between the original visually exposed track orientation and the line orthogonal to it. There was also significantly less transfer to intermediate targets lying between the original track orientation and the orthogonal in the opposite direction. The reason for this curvilinear pattern could not be explained satisfactorily. However, McIntyre and Pick demonstrated that this adaptation and transfer was motoric and/or proprioceptive in nature, rather than visual.

In the present study, the organization of perceptualmotor space was examined again by studying adaptation to a 90-deg rotation of the perceptual-motor field. However, now adaptation was compared when the subject's performance was restricted to either one or two dimensions during exposure. Of central interest were both the amount and the pattern of the adaptation transferred between the exposed and the nonexposed dimensions. Distinct outcomes were expected, given each of the different conceptualizations of perceptualmotor space. For example, the assumption that perceptual-motor space consists of an integrated system led to the expectation that the amount of adaptation generated from manual exposure to either one or two dimensions during visual rotation would be equivalent and would transfer fairly equivalently across nonexposed 
dimensions. In contrast, the assumption that perceptualmotor space involves a system of independent dimensions led to the expectation that the amount of adaptation generated from exposure to either one or two dimensions during visual rotation would be equivalent and would not transfer to nonexposed dimensions. And, finally, given McIntyre and Pick's (1974) results and assuming some transfer of adaptation from the exposed to the nonexposed dimension led to the expectation that the amount of adaptation generated from exposure to two dimensions would reflect an algebraic combination of the adaptation associated with each dimension exposed individually. That is, the transferred pattern of adaptation obtained would reflect an algebraic combination of the transferred patterns of adaptation associated with each dimension. These expectations were examined in the present study.

\section{METHOD}

\section{Subjects}

Forty-eight right-handed volunteers with normal or correctedto-normal vision were recruited from an introductory psychology course. All subjects were naive with respect to the purpose of the experiment.

\section{Apparatus}

The basic apparatus is the same as that used by McIntyre and Pick (1974) and is depicted in Figure 1 . The physical arrangement (following a suggestion by Howard \& Templeton, 1966) allowed the subject to see his or her own hand in approximately its true location by means of a TV monitor. Rotation of the visual field was produced by rotating the TV camera 90 deg about its lens axis. This technique is similar in principle to that devised by Smith and Smith (1962).

During the exposure period, a stationary track formed by a $.75-\mathrm{cm}$ interspace between the edges of two metal plates was located directly beneath the subject's hand. This stationary track consisted of two $20-\mathrm{cm}$ legs that met to form a right angle with its apex in the center of the subject's visual and manual field. The subject's task was to trace along this track with a stylus held in the right hand. The subject was given experience in tracing either along the $20-\mathrm{cm}$ length of one of the legs (either the horizontal or the vertical leg) of the track or along the entire $40-\mathrm{cm}$ length of both legs (horizontal and vertical) of the right angle formed by the track. A metronome was used to help

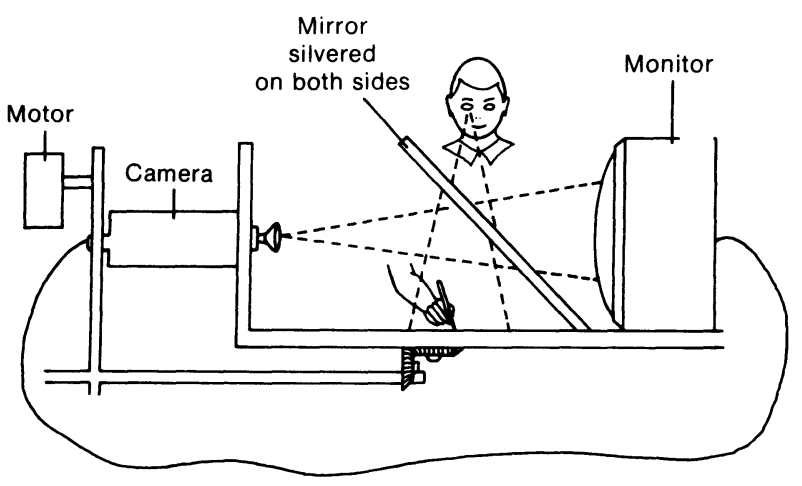

Figure 1. The basic apparatus.

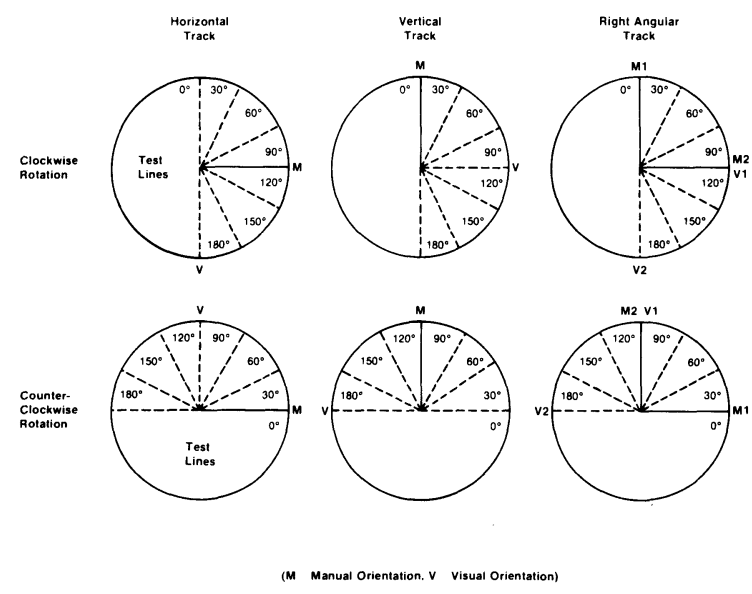

Figure 2. Conditions and test lines.

maintain a pace of about $20 \mathrm{~cm}$ (one leg) every $3 \mathrm{sec}$. Contact between the stylus and the edge of the track closed an electrical circuit, which resulted in the clearly audible clicking of a counter.

Both before and after the exposure period, the experimenter blocked the subject's view of his or her own hand by interposing a white background containing an adjustable test line. Rotation of the test line about its midpoint (which was coincident with the TV camera's lens axis) allowed the experimenter to present any of seven test lines. The orientations of the test lines differed by some multiple (between 0 and 6 ) of $30 \mathrm{deg}$ from the orientation of the exposure track. The subject traced, with his or her (right) unseen hand, each test line onto a large pad of paper that replaced the stationary track. Viewing was binocular throughout the exposure and the tests.

\section{Design and Procedure}

The experiment was conducted in three phases. During the first phase, the subject's view of his or her hand was blocked by the white background containing the variable test line. The task was to copy each of the seven test lines presented in a random order. The test lines differed in their orientations, which were determined in part by the drawing movements made by the subject. The subject was instructed to begin his or her line at the end designated by a small dot, that is, at an enlargement of the line. For example, a horizontal line drawn from left (where the dot was located) to right was distinct from a horizontal line drawn from right to left.

During the second phase, the subject could see his or her hand on the TV monitor as reflected by the mirror. The task was to trace with a stylus back and forth for $\mathbf{1 5}$ min along a stationary track that was either linear or right-angular. Contact between the stylus and the edge of the track produced a counter-clicking noise, which the experimenter defined as an error. The subject was encouraged to trace the linear track once every $3 \mathrm{sec}$ (or the right-angular track once every $6 \mathrm{sec}$ ) with a minimum number of errors.

Eight subjects were assigned randomly to each of six exposure conditions ${ }^{1}$ defined both by the direction of the $90-\mathrm{deg}$ rotation of the visual field (either clockwise or counterclockwise) and by the track that they were asked to trace (eight horizontal, vertical, or both-i.e., right angular). Each of the exposure conditions and its test lines are presented in Figure 2.

During the third phase, the subject's view of his or her hand was blocked again. The subject's task was again to copy each of the seven test lines. The procedure was identical to that used during the first phase. 


\section{RESULTS AND DISCUSSION}

The basic data were the amounts of adaptation revealed by comparison of the directions of the test lines drawn before exposure to the rotation of the visual field with those drawn after exposure. ${ }^{2}$ Generalized adaptation was measured by the changes in the drawings of the test lines with visual orientations different from the orientation of the exposure track. For purposes of analysis, all differences in the adaptive direction between test lines drawn before and after exposure were assigned positive values, whereas differences in the opposite direction were assigned negative values. Application of a 2 (rotation) $\times 3$ (track) $\times 7$ (test line) analysis of variance to these adaptation values revealed a significant effect for the track $[F(2,42)=10.06, p<.01]$ and a significant rotation $\mathrm{x}$ track $\mathrm{x}$ test line interaction $[F(12,252)=5.15, p<.01]$, but no significant effect for the direction of visual rotation or for the orientation of the test lines and no other significant interactions. The mean adaptations associated with each test line under each exposure condition are presented in Table 1.

Newman-Keuls multiple comparison tests applied to the mean adaptations obtained for the three track conditions revealed that the mean adaptation for the rightangular track, $13.01 \mathrm{deg}$, was significantly greater $(p<.01)$ than that obtained for either the horizontal or the vertical track alone, 8.01 and $6.07 \mathrm{deg}$, respectively. Evidently, the mean adaptation generated by experience with two directions of manual movement during exposure to the 90-deg visual rotation is much greater than that generated by experience with only one direction of manual movement during exposure to this visual rotation.

Inspection of Table 1 reveals a pattern of adaptation similar to that found by McIntyre and Pick (1974) for the horizontal and vertical tracks; that is, the amount of adaptation obtained varied in a curvilinear pattern as a function of the difference beween the exposure-track direction and the test-line direction. For example, combining the data for the clockwise rotation $\mathrm{x}$ vertical track (CW-V) condition and the counterclockwise rota- tion $\mathrm{x}$ horizontal track $(\mathrm{CCW}-\mathrm{H})$ condition, as is done in Table 2, revealed that the primary adaptation (the test line labeled $90 \mathrm{deg}$ ) was sometimes greater and sometimes less than the generalized adaptation. (Note that, for these two conditions, the label of each test line reflects its departure from the manual orientation of the track in the direction of the visual rotation.) More specifically, the generalized adaptation associated with the test lines labeled 30 and 60 deg was greater than the primary adaptation, and the generalized adaptation associated with the test lines labeled 120 and $150 \mathrm{deg}$ was less than the primary adaptation. Next, combining the data for the clockwise rotation $\mathrm{x}$ horizontal track (CW-H) condition and the counterclockwise rotation $\mathrm{x}$ vertical track (CCW-V) condition revealed a similar pattern of results for the primary versus generalized adaptation, with the phase of this sinusoidal pattern offset by $90 \mathrm{deg}$. (Note that, for these two conditions, the label of each test line reflects its departure plus $90 \mathrm{deg}$ from the manual orientation of the track in the direction of the visual rotation.) More specifically, here, the generalized adaptation associated with the test lines labeled 120 and $150 \mathrm{deg}$ was greater than the primary adaptation, and the generalized adaptation associated with the test lines labeled 30 and 60 deg was less than the primary adaptation. As noted earlier, these results are very similar to McIntyre and Pick's results, which had indicated that adaptation transferred from the exposure orientation to other orientations and that the transfer was both greatest and least for orientations to which the subjects had not been exposed during the distortion.

Table 1 shows that the pattern of adaptation found for the right-angular track was different from that reported by McIntyre and Pick (1974); in the present study, the primary adaptation was usually less than the generalized adaptation. More specifically, the generalized adaptation associated with the test lines labeled $30,60,120$, and $150 \mathrm{deg}$ was greater than the primary adaptation. A likely explanation for this difference in results lies in the adaptation associated with the rightangular track's legs' being 90 deg out of phase with each other. The resulting sinusoidal patterns of transferred

Table 1

Mean Adaptation (in Degrees)

\begin{tabular}{|c|c|c|c|c|c|c|}
\hline \multirow[b]{4}{*}{ Test Line* } & \multicolumn{6}{|c|}{ Direction of Visual Rotation } \\
\hline & \multicolumn{3}{|c|}{ Clockwise } & \multicolumn{3}{|c|}{ Counterclockwise } \\
\hline & \multicolumn{3}{|c|}{ Track } & \multicolumn{3}{|c|}{ Track } \\
\hline & Horizontal & Vertical & Right Angular & Horizontal & Vertical & Right Angular \\
\hline 0 & 10.13 & 2.13 & 10.63 & 5.63 & 7.13 & 9.25 \\
\hline 30 & 3.25 & 6.00 & 20.38 & 11.25 & 6.50 & 11.63 \\
\hline 60 & 4.88 & 4.50 & 11.13 & 14.13 & 6.00 & 12.88 \\
\hline 90 & 7.13 & 6.63 & 9.75 & 8.75 & 10.38 & 10.25 \\
\hline 120 & 14.25 & 0.50 & 16.50 & 4.63 & 14.25 & 14.88 \\
\hline 150 & 10.25 & 1.00 & 14.25 & -0.13 & 9.88 & 14.50 \\
\hline 180 & 9.88 & 3.00 & 13.38 & 8.13 & 7.13 & 12.75 \\
\hline
\end{tabular}

*In degrees. 
Table 2

Predicted and Obtained Mean Adaptations (in Degrees)

\begin{tabular}{|c|c|c|c|c|c|}
\hline \multirow[b]{2}{*}{ Test Line } & \multicolumn{2}{|c|}{ Conditions } & \multirow[b]{2}{*}{ Predicted } & \multirow[b]{2}{*}{ Obtained } & \multirow[b]{2}{*}{ Difference } \\
\hline & $\begin{array}{c}\text { Mean } \\
(\mathrm{CW}-\mathrm{V}+\mathrm{CCW}-\mathrm{H}) \\
\end{array}$ & $\begin{array}{c}\text { Mean } \\
(\mathrm{CW}-\mathrm{H}+\mathrm{CCW}-\mathrm{V}) \\
\end{array}$ & & & \\
\hline 0 & 3.88 & 8.63 & 12.51 & 9.94 & -2.57 \\
\hline 30 & 8.63 & 4.88 & 13.51 & 16.00 & 2.49 \\
\hline 60 & 9.32 & 5.44 & 14.76 & 12.00 & -2.76 \\
\hline 90 & 7.69 & 8.76 & 16.45 & 10.00 & -6.45 \\
\hline 120 & 2.57 & 14.25 & 16.82 & 15.69 & -1.13 \\
\hline 150 & .44 & 10.07 & 10.51 & 14.38 & 3.87 \\
\hline 180 & 5.57 & 8.51 & 14.08 & 13.07 & -1.01 \\
\hline Mean & 5.44 & 8.65 & 14.09 & 13.01 & -1.08 \\
\hline
\end{tabular}

*In degrees.

adaptation associated with each leg combine algebraically and level each other out. If it is assumed that the overall adaptation obtained for the right-angular track reflects separate contributions from each leg, the best estimates of the contribution of each leg can be achieved by using the adaptation values obtained on the horizontal- and vertical-track-only conditions. These adaptation values can then be combined to generate predicted adaptation values for the right-angular track (see Table 2). Comparison of these predicted adaptations with those actually obtained revealed the average difference to be only $-1.08 \mathrm{deg}(99 \%$ confidence bounds $= \pm 4.55)$, a difference that did not depart significantly from zero when the predicted adaptation was compared with the adaptation actually obtained for each subject $[\mathrm{t}(15)=.77$, $\mathrm{p}>.05]$.

Although the pattern of adaptation found for the right-angular track in the present study differs from that reported by McIntyre and Pick (1974), both patterns indicate that adaptation transfers from the exposure dimensions to other dimensions and that the transfer is greatest for dimensions to which the subject has not been exposed during the distortion. With respect to the structure of perceptual-motor space, the results of the present study indicate that neither the conceptualization of perceptual-motor space as an integrated system of dimensions nor the conceptualization of it as a system of independent dimensions is correct. The asymmetric pattern of adaptation observed eliminates the former, whereas the transfer of adaptation to nonexposed dimensions observed for all conditions eliminates the latter. Rather, as suggested by McIntyre and Pick, the structure of perceptual-motor space is something between these two extremes.

\section{REFERENCES}

Howard, I. P., \& Templeton,- W. B. (1966). Human spatial orientation. London: Wiley.

McInTyre, C. W., \& Pick, H. L., Jr. (1974). Transfer of adaptation to rotation of the visual field. Journal of Experimental Psychology, 103, 782-791.

Pick, H. L., Jr., Warken, D. H., McIntyre, C. W., \& Appel, L. (1972). Transfer and the organization of perceptual-motor space. Psychologische Forschung, 35, 163-177.

Sмiтн, K. U., \& Sмiтh, W. M. (1962). Perception and motion. Philadelphia: Saunders.

\section{NOTES}

1. Typically, a within-subjects design would be used in this type of study. Unfortunately, because little is known as yet about the effects of repeated exposures to the visual rotations used here, the authors elected to use a potentially "less sensitive" between-subjects design rather than risk possible cumulative exposure effects. The results indicate that the between-subjects design used was of sufficient sensitivity.

2 . Because the tracing of a test line usually did not result in a perfectly straight line, the angle of a test line was measured with reference to the straight line joining the end points of the test-line tracing.

(Manuscript received for publication September 22, 1983.) 